\title{
Une initiative pédagogique autour du kart électrique : le projet
}

\section{collectif intensif}

\author{
Sébastien Jacques ${ }^{1,2}$, Thierry Lequeu ${ }^{3,4}$, Valéry Dewancker ${ }^{5}$
}

${ }^{1}$ Polytech Tours, Département Électronique et Énergie, 7 avenue Marcel Dassault, 37200 Tours

${ }^{2}$ GREMAN CNRS UMR 7347, 7 avenue Marcel Dassault, 37200 Tours

${ }^{3}$ IUT de Tours, département GEII, avenue Monge, 37200 Tours

${ }^{4}$ Association e-Kart, 152 avenue de Grandmont, 37550 Saint-Avertin

${ }^{5}$ Kart Masters, 9 rue Saint Denis, 72300 Sablé-sur-Sarthe

Téléphone : +33(0)2 473613 38. Courriel : sebastien.jacques@univ-tours.fr

\section{Résumé}

Cet article propose un retour d'expériences d'une nouvelle approche pédagogique de l'enseignement de l'électronique et du génie électrique menée au sein de Polytech Tours (école d'ingénieurs). Cette approche est fondée sur un projet intensif (trois semaines consécutives à plein temps dédiées à l'étude et à la réalisation d'un système industriel) réalisée de manière collective (33 élèves ingénieurs répartis en 3 groupes de 11 étudiants). L'étude a consisté à procéder à l'électrification d'un châssis de kart et à rendre le véhicule électrique performant. Les élèves ingénieurs ont entre autre justifié tous les éléments composant le système de motorisation, assemblé toutes les pièces du kart électrique, testé le bon fonctionnement de ce dernier et apporté une innovation technologique autour de la récupération d'énergie cinétique au freinage par l'intermédiaire de supercondensateurs. Cette initiative, fortement appréciée des étudiants puisque chacun d'entre eux a joué un rôle actif dans la réalisation du projet, a en outre été saluée par la presse locale et régionale.

\section{Mots-clés}

Innovation pédagogique, projet collectif intensif, kart électrique. 


\section{Introduction}

Depuis quelques années, la voiture électrique séduit de nombreux usagers pour de nombreuses raisons, qu'elles soient technicoéconomiques, environnementales et sociales [1-5]. Le moteur électrique est entre autre un substitut incontestable au moteur thermique. En effet, dans son analyse du cycle de vie parue en 2013, l'agence de l'environnement et de la maîtrise de l'énergie (ADEME) a estimé la contribution climatique globale du véhicule électrique à 9 tonnes d'équivalent $\mathrm{CO}_{2}$ sur l'ensemble de sa durée de vie contre 22 tonnes pour un véhicule thermique [6]. Même si la lutte contre le réchauffement climatique demeure au cœur des préoccupations politiques sur les plans national et international, la voiture électrique, compte-tenu de son autonomie, doit inciter chaque individu à réfléchir sur ses propres usages afin d'optimiser ses déplacements.

Dans un récent communiqué, l'association nationale pour le développement de la mobilité électrique (AVERE France) a mis en exergue qu'environ 110000 véhicules électriques ont été immatriculés dans le monde au cours du premier semestre 2015 [7]. L’Europe représente le premier marché mondial devant les États-Unis. La Norvège se place largement en tête du marché européen (14 024 nouvelles immatriculations) [8]. La France confirme sa montée en puissance (10 063 immatriculations de modèles électriques particuliers et utilitaires). Elle se situe au deuxième rang européen devant le Royaume-Uni (5 103 véhicules électriques immatriculés) et l'Allemagne (4 710). Dans le palmarès des marques les plus vendues, Nissan est au premier plan sur le marché européen (environ 38\% des véhicules électriques nouvellement immatriculés en 2015) [9]. Aux États-Unis, Tesla, avec 45\% des immatriculations, est de loin la marque incontournable [10].

Malgré ce contexte, la voiture électrique est très loin de remplacer définitivement le véhicule thermique. En France, elle représente moins de 1\% du parc automobile. En 2011, Carlos Ghosn, l'actuel PDG de l'alliance Renault-Nissan BV, imaginait vendre 1,5 million de modèles électriques en 2016 [11]. Deux raisons principales permettent d'expliquer la non-justesse de ces prévisions : le prix élevé et la faible autonomie des batteries. Par exemple, un des véhicules phare de la marque Nissan est accessible à partir de 17000 Euros (hors location des batteries) pour une autonomie spécifiée à près de $200 \mathrm{~km}$ dans le cadre du nouveau cycle européen de conduite (ou NEDC de l'acronyme anglophone New European Driving Cycle). 
À la suite de la COP21 qui s'est tenue à Paris du 30 novembre 2015 au 11 décembre 2015, Ségolène Royal, Ministre de l'Écologie, du Développement durable et de l’Énergie depuis 2014, a déclaré vouloir « lancer un appel à projet mondial pour la conception, le développement et la commercialisation d'un véhicule électrique populaire à moins de 7000 dollars » [12]. L'enjeu est de taille, voire impossible pour de nombreux constructeurs automobiles. À ce jour, il est important de donner aux futurs cadres de demain tous les moyens nécessaires pour répondre aux défis technicoéconomiques, sociaux et environnementaux en vue du développement massif du véhicule électrique. Pour cela, les programmes pédagogiques des domaines de l'électronique et du génie électrique doivent nécessairement être adaptés.

L'école Polytechnique de l'Université de Tours (Polytech Tours, école d'ingénieurs), représentée par son Département Électronique et Énergie (DEE), a initié une nouvelle approche d'enseignement des sciences de l'ingénieur autour du véhicule électrique : le projet collectif intensif, en utilisant un support que représente le kart électrique. Ce modèle réduit de voiture électrique a démontré depuis de nombreuses années son caractère pédagogique pluridisciplinaire dans la mesure où il suscite l'apprentissage de savoirs (conception mécanique, systèmes électroniques embarqués, conversion et gestion de l'énergie électrique) [13-16].

Dans le cadre de ce projet intitulé « La course à l'électrification des véhicules », réalisé durant trois semaines consécutives (56 heures encadrées), trois équipes, composées chacune de 11 élèves ingénieurs de 4 ème année de la spécialité «Électronique et Systèmes de l'Énergie Électrique », ont dû :

- Procéder à l'électrification d'un châssis de kart en respectant un cahier des charges fonctionnel. Le véhicule doit montrer un niveau significatif de performance.

- Étudier et réaliser une fonction électronique embarquée dans le véhicule (par exemple, indication de l'autonomie des batteries, réalisation d'un chronomètre ou d'un compteur de vitesse, suivi à distance de l'état fonctionnel du véhicule, ...).

Ce projet est le fruit d'une collaboration entre plusieurs partenaires académiques - le département Génie Électrique et Informatique Industrielle (GEII) de l'IUT de Tours, ainsi que Polytech Tours -, l'association e-kart et la société Kart Masters de Sablé-sur-Sarthe. 
Cet article vise d'une part, à présenter la méthodologie et les résultats obtenus par les élèves ingénieurs à l'issue de ce projet collectif intensif et d'autre part, d'apporter un retour d'expériences en termes d'évaluation des connaissances et des compétences.

Dans un premier temps, les caractéristiques techniques du kart électrique seront explicitées. Ensuite, les principales réalisations menées par les élèves ingénieurs seront détaillées. La communication autour de l'activité «e-karting » sera en particulier mise en exergue. Enfin, l'évaluation des connaissances et des compétences acquises par les étudiants sera discutée. Cette initiative pédagogique a également été évaluée par les élèves ingénieurs. Une synthèse des résultats de cette évaluation sera présentée à la fin de cet article.

\section{Analyse fonctionnelle du kart électrique}

\subsection{Chaîne de conversion d'énergie}

La figure 1 illustre la chaîne énergétique à l'origine de la mise en mouvement du kart. Elle est composée d'une partie dédiée à la conversion et à la gestion de l'énergie électrique et d'une transmission mécanique. La source d'énergie électrique est fournie par quatre batteries (technologie : Plomb) de $12 \mathrm{~V}$ associées en série, ayant chacune une capacité de 38 Ah. Chaque batterie dispose d'un chargeur d'entretien de type «CTEK MXS 7.0 12 V 7 A » connecté au réseau électrique de distribution (230 V AC RMS, 50 Hz).

La source d'énergie électrique continue de $48 \mathrm{~V}$ est en charge d'alimenter un moteur synchrone triphasé de type «ME1304 PMSM Brushless ». Ce moteur, qui peut être alimenté entre $24 \mathrm{~V}$ et 72 V, fournit une puissance de sortie de $8,5 \mathrm{~kW}$ à $72 \mathrm{~V}$. Sa vitesse de rotation peut atteindre $4000 \mathrm{tr} / \mathrm{min}$.

Pour faire le lien entre les batteries et le moteur, un contrôleur électronique triphasé de type « SEVCON GEN4 G4845 » est utilisé. Il peut délivrer, à la tension nominale de $48 \mathrm{~V}$, un courant continu de $180 \mathrm{~A}$ (pendant 60 min) et un courant maximal de $450 \mathrm{~A}$ (pendant $2 \mathrm{~min}$ ). Le contrôleur doit à la fois interpréter la consigne de la pédale d'accélérateur et commander le moteur triphasé c'est-à-dire ajuster sa vitesse et son couple.

Concernant la transmission mécanique, deux poulies sont fixées : la première sur l'axe du moteur électrique et la seconde, sur l'arbre de transmission. Une courroie permet de relier le tout pour récupérer l'énergie du moteur électrique et permettre l'entraînement des deux roues arrière fixées à chaque extrémité de l'arbre de transmission 
rigide (le véhicule ne dispose pas de différentiel mécanique).

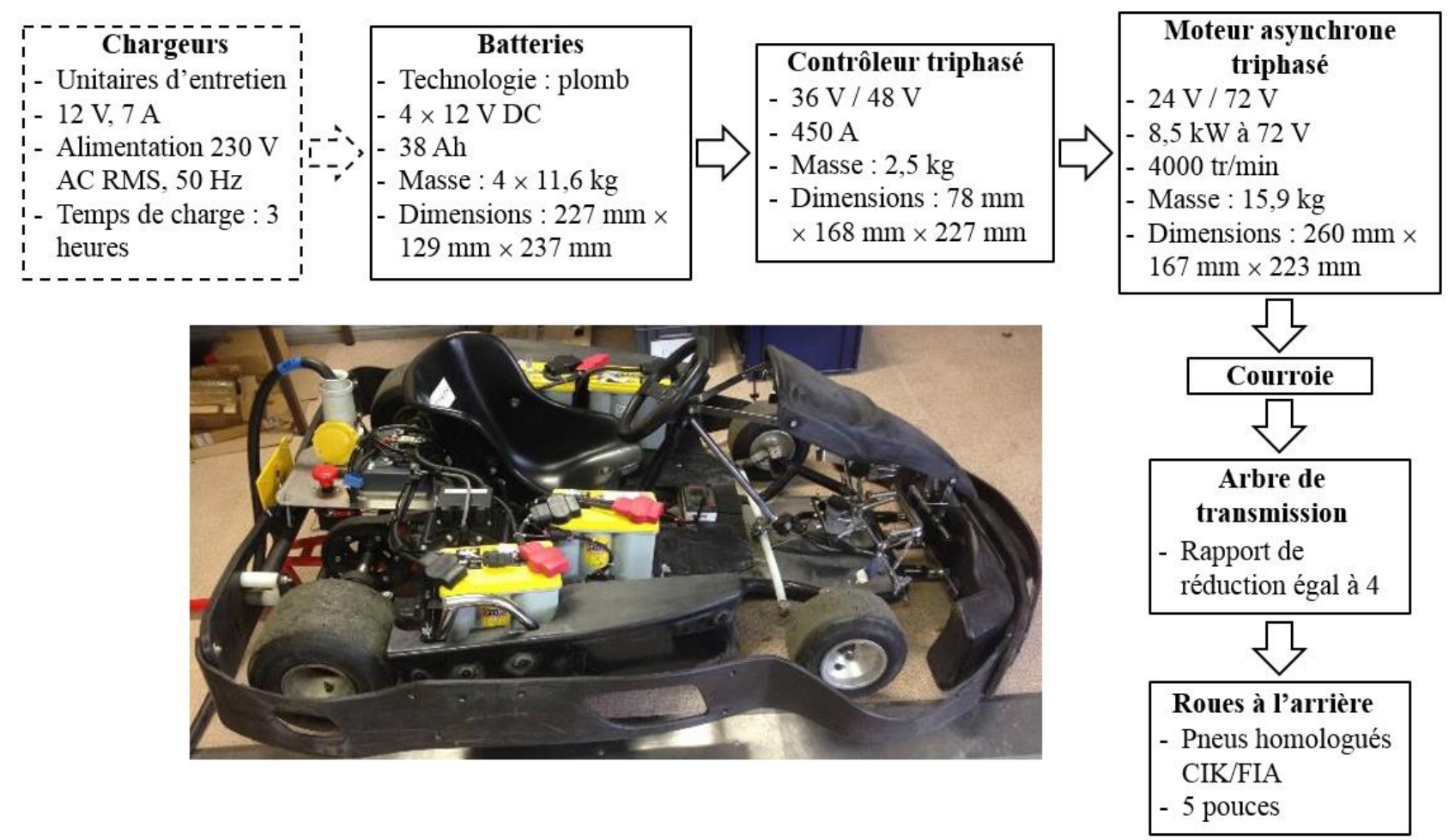

Figure 1 : Chaîne de conversion d'énergie du kart électrique.

\subsection{Justification technique des choix pour le système de motorisation}

\subsubsection{Batteries au plomb $12 \mathrm{~V}, 38 \mathrm{Ah}$}

Le moteur, par l'intermédiaire de son contrôleur, est alimenté en 48 V DC. Quatre batteries de 12 V associées en série sont utilisées. À partir du tableau 1, la technologie au plomb a été choisie principalement pour des raisons de coût en dépit de sa faible énergie massique (entre $20 \mathrm{Wh} / \mathrm{kg}$ et $40 \mathrm{Wh} / \mathrm{kg}$ ) ou énergie volumique (entre $40 \mathrm{Wh} / \mathrm{kg}$ et $100 \mathrm{Wh} / \mathrm{L}$ ). Il est important de noter des batteries du fabricant OPTIMA ${ }^{\circledR}$ sont utilisées. D’un point de vue opérationnel, A. Sivert et al. ont démontré dans leur ouvrage qu'une énergie de $833 \mathrm{Wh}$ est nécessaire pour garantir un fonctionnement du kart à $5 \mathrm{~kW}$, sous $48 \mathrm{~V} \mathrm{DC}$, pendant 10 minutes [17]. En théorie, une batterie d'une capacité au moins égale à 17,4 Ah pourrait alors convenir. Dans le cadre de cette réalisation, des batteries de capacité de 38 Ah ont été retenues. Ces batteries répondent au besoin de fonctionnement du véhicule électrique. De plus, leur masse $(11,6 \mathrm{~kg})$ est inférieure à celle des batteries de $48 \mathrm{Ah}(16,6 \mathrm{~kg})$ habituellement rencontrées 
dans les karts électriques.

Les batteries au plomb du fabricant OPTIMA ${ }^{\circledR}$ utilisent la technologie SpiralCell ${ }^{\circledR}$ qui permet de combiner les avantages de la batterie de démarrage (délivrer un courant de forte intensité pendant un temps très bref) à ceux de la batterie à décharge profonde (conçue pour donner sa puissance sur une longue durée). En définitive, les batteries choisies sont en mesure de supporter des cycles de charge et de décharge élevés (quelques centaines de cycles) tout en conservant un pourcentage élevé de leur capacité totale d'origine. Contrairement à des batteries au plomb conventionnelles, les batteries OPTIMA ${ }^{\circledR}$ offre une sécurité accrue. L'électrolyte est en outre absorbé dans des séparateurs en fibre de verre microporeuse. Cela permet d'éliminer le risque de fuite d'acide.

\begin{tabular}{|c|c|c|c|c|c|}
\hline Technologie & $\begin{array}{c}\text { Masse (36 V, } \\
10 \mathrm{Ah}) \\
\end{array}$ & $\begin{array}{c}\text { Nombre de } \\
\text { cycles }\end{array}$ & $\begin{array}{c}\text { Prix (36 V, } \\
10 \mathrm{Ah}) \\
\end{array}$ & Avantages & Inconvénients \\
\hline $\begin{array}{l}\text { Lithium - } \\
\text { Polymère (Li- } \\
\text { Po) }\end{array}$ & $3 \mathrm{~kg}$ & $\begin{array}{c}\text { De } 1000 \text { à } \\
2000\end{array}$ & 600 Euros & $\begin{array}{c}\text { Légère, pas } \\
\text { d'effet } \\
\text { mémoire, peu } \\
\text { d'autodécharge }\end{array}$ & Prix \\
\hline $\begin{array}{l}\text { Lithium - ion } \\
\text { (Li-ion) }\end{array}$ & $4 \mathrm{~kg}$ & $\begin{array}{c}\text { De } 1000 \text { à } \\
1500\end{array}$ & 400 Euros & $\begin{array}{c}\text { Légère, pas } \\
\text { d'effet } \\
\text { mémoire, peu } \\
\text { d'autodécharge }\end{array}$ & $\begin{array}{l}\text { Prix, risque } \\
\text { d'incendie }\end{array}$ \\
\hline $\begin{array}{l}\text { Lithium fer - } \\
\text { phosphate } \\
\text { (LiFePO4) }\end{array}$ & $5 \mathrm{~kg}$ & $\begin{array}{l}\text { Jusqu'à } \\
3000\end{array}$ & 500 Euros & $\begin{array}{l}\text { Légère, } \\
\text { recharge très } \\
\text { rapide, pas } \\
\text { d'effet } \\
\text { mémoire }\end{array}$ & $\begin{array}{l}\text { Technologie } \\
\text { récente, prix }\end{array}$ \\
\hline $\begin{array}{l}\text { Nickel - métal } \\
\text { hydrure } \\
(\mathrm{NiMH})\end{array}$ & $5 \mathrm{~kg}$ & $\begin{array}{l}\text { De } 500 \text { à } \\
1000\end{array}$ & 300 Euros & $\begin{array}{l}\text { Rapport poids } \\
\text { / puissance / } \\
\text { prix }\end{array}$ & $\begin{array}{c}\text { Autodécharge, } \\
\text { Vieillissement, } \\
\text { effet mémoire, } \\
\text { sensible aux } \\
\text { variations de } \\
\text { température }\end{array}$ \\
\hline Plomb & $10 \mathrm{~kg}$ & $\begin{array}{c}\text { De } 300 \text { à } \\
500\end{array}$ & 150 Euros & $\begin{array}{l}\text { Prix, pas } \\
\text { d'effet } \\
\text { mémoire, } \\
\text { durée de vie, } \\
\text { recyclable }\end{array}$ & $\begin{array}{c}\text { Poids, volume, } \\
\text { sensible au } \\
\text { froid, charge } \\
\text { lente, } \\
\text { autodécharge }\end{array}$ \\
\hline
\end{tabular}

Tableau 1 : Justification technique du choix des batteries au plomb de $12 \mathrm{~V}, 38 \mathrm{Ah}$ [18]. 


\subsubsection{Contrôleur triphasé « SEVCON GEN4 G4845 »}

Le contrôleur triphasé de type « SEVCON GEN4 G4845 » (cf. Figure 2) est un onduleur triphasé basse tension et fort courant. Il est composé de trois boucles permettant de contrôler la vitesse, le courant (donc le couple) et la tension. Dans cette application, le contrôleur est piloté en couple et intègre une limitation de la vitesse du moteur. La consigne du couple est donnée par le pilote via la pédale d'accélérateur. Les instructions du courant et de la tension, quant à elles, sont transmises au contrôleur par l'intermédiaire du logiciel interne du contrôleur. Ce logiciel de paramétrage appelé «DVT», fourni par le constructeur, permet à l'utilisateur de régler le couple maximal du moteur, la rampe d'accélération, la limitation de courant que l'on souhaite appliquer, ou encore la vitesse maximale désirée en marche avant et en marche arrière du moteur.

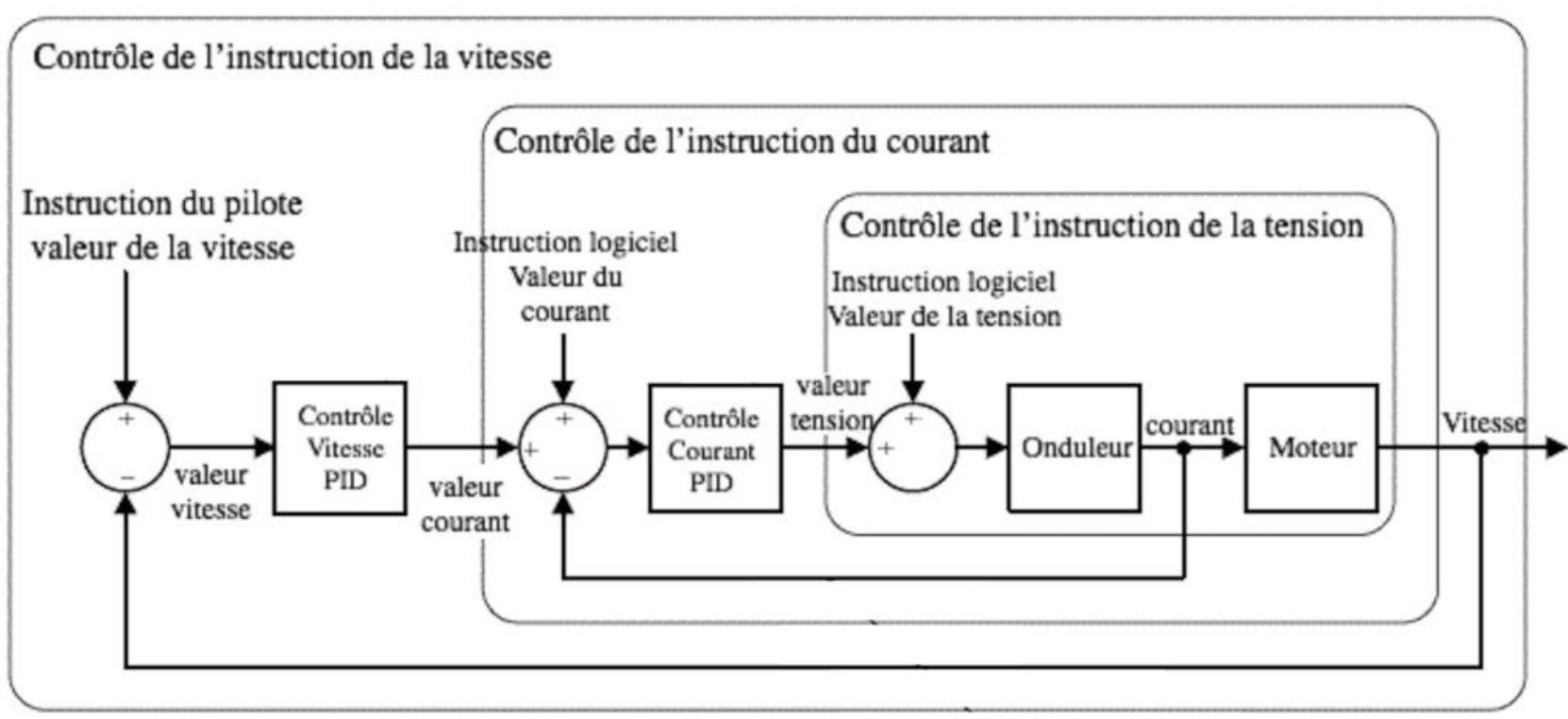

Figure 2 : Synoptique du contrôleur triphasé de type «SEVCON GEN4 G4845 ».

D'après le synoptique de la figure 2, le moteur est piloté par un onduleur triphasé. La documentation technique du contrôleur de type «SEVCON GEN4 G4845 » indique que ce dernier est constitué de 6 interrupteurs de puissance de « dernière génération ». Leur fréquence de commande est comprise entre $16 \mathrm{kHz}$ et $24 \mathrm{kHz}$. Compte-tenu de la puissance de sortie $(8,5 \mathrm{~kW}$ sous $48 \mathrm{~V})$, on peut supposer que ces transistors sont à grille isolée (MOSFET ou IGBT) et fabriqués sur un substrat à grand gap, par exemple le carbure de silicium (SiC). Toutefois, ces informations ne sont pas indiquées dans la documentation technique du fabricant. Si l'on part de l'hypothèse de 
l'utilisation de 6 MOSFETs de type « NDPL180N10B » $\left(\mathrm{V}_{\mathrm{DSS}}=100 \mathrm{~V}, \mathrm{R}_{\mathrm{DS}(\mathrm{ON})}=3 \mathrm{~m} \Omega, \mathrm{I}_{\mathrm{D}(\mathrm{MAX})}=180 \mathrm{~A}\right.$, boîtier TO-220AB) du constructeur « ON Semiconductor », chacun d'entre eux étant par exemple piloté à la fréquence de $20 \mathrm{kHz}$, le tableau 2 illustre la répartition des pertes en conduction en fonction du rapport cyclique de la commande. Pour simplifier l'étude, la forme d'onde du courant circulant dans le drain d'un MOSFET est supposée rectangulaire d'amplitude environ égale à $I_{D(M A X)}(8,5 \mathrm{~kW} / 48 \mathrm{~V})$. L'équation 1 donne l'expression littérale des pertes en conduction $\left(P_{O N}\right)$ en fonction de la $R_{D S(O N)}$, l'intensité du courant efficace $\left(I_{D(R M S)}\right)$ circulant dans le drain du transistor et le rapport cyclique $(\alpha)$ de la commande. La valeur de la résistance $R_{D S(O N)}$ est prise dans le cas le plus défavorable (environ 5,5 $\mathrm{m} \Omega$ ) c'est-à-dire à la valeur maximale de la température de jonction $\left(175^{\circ} \mathrm{C}\right)$ spécifiée dans la documentation technique du constructeur. Le tableau 2 illustre ainsi le fait que plus le temps de conduction d'un MOSFET est important, plus les pertes en conduction sont importantes.

$-P_{O N}:$ pertes en conduction $[\mathrm{W}]$.

$$
P_{O N}=R_{D S(O N)}\left(T_{j}\right) \times I_{D(R M S)}
$$

Équation 1

- $R_{D S(O N)}\left(T_{j}\right)$ : on considère le cas le plus défavorable $\left(5,5 \mathrm{~m} \Omega\right.$ à $\left.175^{\circ} \mathrm{C}\right)$.

$-I_{D(R M S)}^{2}=\alpha \times I_{D(M A X)}^{2}$.

$-\alpha$ : rapport cyclique.

- $I_{D(M A X)}$ : intensité maximale du courant circulant dans le drain du MOSFET.

En utilisant la documentation technique du MOSFET de type «NDPL180N10B », les temps de montée $\left(t_{r}\right)$ et de descente $\left(t_{f}\right)$ du courant valent respectivement $320 \mathrm{~ns}$ et $130 \mathrm{~ns}$. L'équation 2 et l'équation 3 donnent respectivement les expressions littérales des pertes à l'amorçage $\left(P_{\text {amorgage }}\right)$ et au blocage $\left(P_{\text {blocage }}\right)$ du transistor en fonction de la tension de blocage $\left(U_{D S}\right)$, de l'intensité maximale du courant $\left(I_{D(M A X)}\right)$ et de la fréquence de fonctionnement $(F)$. Le tableau 3 met en exergue l'importante de la fréquence de commutation. Plus cette dernière est élevée et plus les pertes en commutation augmentent.

$$
P_{\text {amorçage }}=0,5 \times U_{D S} \times I_{D(M A X)} \times t_{r} \times F
$$

- $P_{\text {amorgage }}$ : pertes à l'amorçage [W].

- $U_{D S}$ : tension entre le drain et la source [V]. On suppose ici : $U_{D S}=48 \mathrm{~V}$.

- $t_{r}:$ temps de montée [s]. On suppose ici : $t_{r}=320 \mathrm{~ns}$.

- $F$ : fréquence de fonctionnement $[\mathrm{Hz}]$.

- $P_{\text {blocage }}:$ pertes au blocage [W].

$$
P_{\text {blocage }}=0,5 \times U_{D S} \times I_{D(M A X)} \times t_{f} \times F
$$

- $U_{D S}$ : tension entre le drain et la source [V]. On suppose ici : $U_{D S}=48 \mathrm{~V}$.

$-t_{f}$ : temps de montée [s]. On suppose ici : $t_{f}=130 \mathrm{~ns}$.

- $F$ : fréquence de fonctionnement $[\mathrm{Hz}]$. 


\begin{tabular}{ccccccc}
\hline $\begin{array}{c}\text { Rapport cyclique du } \\
\text { MOSFET }\end{array}$ & $\begin{array}{c}0,1 \mathrm{~T}= \\
\text { NDPL180N10B } \text { » piloté } \\
\text { à 20 kHz }\end{array}$ & $\begin{array}{c}0,2 \mathrm{~T}= \\
10 \mu \mathrm{s}\end{array}$ & $\begin{array}{c}0,3 \mathrm{~T}= \\
15 \mu \mathrm{s}\end{array}$ & $\begin{array}{c}0,5 \mathrm{~T}= \\
25 \mu \mathrm{s}\end{array}$ & $\begin{array}{c}0,7 \mathrm{~T}= \\
35 \mu \mathrm{s}\end{array}$ & $\begin{array}{c}0,9 \mathrm{~T}= \\
45 \mu \mathrm{s}\end{array}$ \\
\hline $\begin{array}{c}\text { «ertes en conduction (W) } \\
\text { N }\end{array}$ & 17,8 & 35,6 & 53,5 & 89,1 & 124,7 & 160,4 \\
\hline
\end{tabular}

Tableau 2 : Estimation des pertes dues à la conduction de chaque interrupteur actif (onduleur utilisé dans le contrôleur de type «SEVCON GEN4 G4845 ») en fonction du rapport cyclique.

\begin{tabular}{lccccc}
\hline $\begin{array}{c}\text { Fréquence de } \\
\text { fonctionnement du } \\
\text { MOSFET } \\
\text { «NDPL180N10B » }\end{array}$ & $16 \mathrm{kHz}$ & $18 \mathrm{kHz}$ & $20 \mathrm{kHz}$ & $22 \mathrm{kHz}$ & $24 \mathrm{kHz}$ \\
\hline Pertes à l'amorçage (W) & 22,1 & 24,9 & 27,6 & 30,4 & 33,2 \\
Pertes au blocage (W) & 9,0 & 10,1 & 11,2 & 12,4 & 13,5 \\
$\begin{array}{l}\text { Pertes totales dues à la } \\
\text { commutation (W) }\end{array}$ & 31,1 & 35,0 & 38,9 & 42,8 & 46,7 \\
\hline
\end{tabular}

Tableau 3 : Estimation des pertes dues à la commutation de chaque interrupteur actif (onduleur utilisé dans le contrôleur de type «SEVCON GEN4 G4845 ») en fonction de la fréquence.

Tous les résultats ci-dessus mettent finalement en évidence le niveau de puissance à dissiper et permettent d'expliquer la présence d'une semelle en aluminium de $388 \mathrm{~cm}^{2}$ de surface et d'épaisseur égale à $1,2 \mathrm{~cm}$, placée à l'arrière du contrôleur, pour des raisons de dissipation de la température.

\subsubsection{Moteur synchrone triphasé « ME1304 PMSM Brushless »}

Le moteur synchrone triphasé de type « ME1304 PMSM Brushless » est composé d'un rotor interne à aimant permanent et d'un stator à trois enroulements bobinés. Ce type de moteur ne contient aucun collecteur tournant et donc pas de balais. Dans ce cas, il est nécessaire de disposer d'un système électronique de commande assurant la commutation du courant dans les enroulements statoriques. C'est ce système électronique qui définit la direction et la force du champ tournant en fonction de l'information donnée par le capteur de position du rotor. Le rôle de l'ensemble composé du capteur et de l'électronique de commande est d'assurer l'autopilotage du moteur, c'est-àdire l'orthogonalité du flux magnétique rotorique par rapport au flux statorique, rôle dévolu à l'ensemble « balais- 
collecteur » dans le cas d'une machine à courant continu.

Comme indiqué dans le tableau 4, à une puissance quasiment équivalente, le moteur synchrone, en comparaison avec son homologue asynchrone, permet d'avoir un encombrement moindre et une masse réduite ; ces deux caractéristiques sont capitales pour la réalisation du kart électrique.

\begin{tabular}{lcc}
\hline & $\begin{array}{c}\text { Moteur asynchrone } \\
\text { SPEEDOMAX }\end{array}$ & $\begin{array}{c}\text { Moteur synchrone « ME0907 } \\
\text { brushless » }\end{array}$ \\
\hline Puissance $(\mathrm{kW})$ & 4,6 & 4,8 \\
Hauteur $(\mathrm{cm})$ & 17,7 & 20,1 \\
Largeur $(\mathrm{cm})$ & 17,7 & 20,1 \\
Longueur $(\mathrm{cm})$ & 35,5 & 14,8 \\
Masse $(\mathrm{kg})$ & 20 & 10 \\
\hline
\end{tabular}

Tableau 4 : Encombrement et masse d'un moteur AC. Comparaison entre un moteur asynchrone et un moteur synchrone à puissance quasiment équivalente.

\section{Retour d'expériences du projet collectif intensif}

\subsection{Rappel du contexte}

Au cours de la quatrième année de la spécialité d'ingénieur appelée «Électronique et Systèmes de l'Énergie Électrique » de Polytech Tours, les étudiants ont l'occasion de participer collectivement à la réalisation d'un projet, à connotation industrielle, durant 56 heures encadrées ; ces heures étant réparties durant trois semaines consécutives. À l'issue de cette expérience, les élèves ingénieurs doivent maîtriser les méthodes et les outils de l'ingénieur relatifs à l'identification, la modélisation et la résolution de problèmes qu'ils soient non familiers et non complètement définis. En particulier, les compétences décrites ci-après sont évaluées :

- Appliquer et développer les concepts de la conversion de l’énergie électrique.

- Acquérir et intégrer les enjeux et les contraintes de la gestion de l'énergie électrique dans un environnement durable.

- Capacité à s'intégrer dans une organisation, à l'animer et à la faire évoluer : engagement et leadership, 
management de projets, maîtrise d'ouvrage, communication avec des spécialistes comme avec des non-spécialistes. - Capacité à s'insérer dans la vie professionnelle, à s'intégrer dans une organisation, à l'animer et à la faire évoluer : exercice de la responsabilité, esprit d'équipe, engagement et leadership, management de projets, maîtrise d'ouvrage, communication avec des spécialistes comme avec des non-spécialistes, voire la gestion d'entreprise innovante.

Au démarrage du projet, il est important de noter que chacun des trois groupes d'étudiants disposait d'un châssis de kart thermique ; le moteur thermique ayant été préalablement démonté. Afin de garantir dans le temps imparti la transformation de chaque châssis en un véhicule électrique, Polytech Tours a procédé à l'achat de kits comprenant l'ensemble du matériel nécessaire à l'électrification des karts. Cette étape est indispensable afin de ne pas être confronté aux délais d'approvisionnement. Grâce au recul de la société Kart Masters, le kit d'électrification est choisi en fonction du budget de l'école d'ingénieurs. Plusieurs kits permettent d'ailleurs d'atteindre différents objectifs de performances. Dans tous les cas, le kit permet la transformation du kart et assure un bon fonctionnement des différents éléments le composant par une bonne compatibilité entre les batteries, le contrôleur et le moteur.

Le suivi journalier des résultats obtenus par les élèves ingénieurs a été effectué en utilisant une plateforme de communication via Internet. Cette plateforme n'a pas été imposée par les enseignants. Quoiqu'il en soit, l'objectif était de pouvoir disposer d'un carnet de bord pour chacun des 3 groupes. Ce carnet était essentiel pour suivre l'évolution du projet et de pouvoir garantir la livraison en temps et en heure des différents livrables. Les connaissances et les compétences acquises par les étudiants ont en particulier été évaluées, à la fin du projet et de façon inédite, par une soutenance théâtralisée. Chaque groupe a choisi un thème particulier et défini un scénario. Chaque scénario a été présenté devant un public composé de tous les étudiants de 4 ème année de la formation d'ingénieur, des enseignants et enseignants chercheurs de Polytech Tours et des professionnels du domaine de l'industrie (électronique et des transports). En ce qui concerne la scénarisation, un groupe a par exemple décidé de mener un jeu de scène sous la forme d'une arrivée d'une course de kart. Les étudiants de Polytech Tours sont arrivés en tête à la fin de la course. Une étudiante, jouant le rôle d'une journaliste, a interrogé chaque élève du 
groupe pour expliquer les bons résultats obtenus durant la course de kart. Pour les enseignants, cette proposition a été particulièrement intéressante, puisque l'aspect «interview » a permis d'évaluer les compétences liées à la communication ; communication pas nécessairement centrée sur un public initié aux problématiques du véhicule électrique. De plus, le fait d'avoir choisir un scénario s'appuyant sur un « retour en arrière », c'est-à-dire permettant d'expliquer le succès des élèves à la fin de la course, a permis au jury d'évaluer les compétences techniques et scientifiques requises pour que le kart soit fonctionnel.

\subsection{Synthèse du travail réalisé par les élèves ingénieurs}

\subsubsection{Dimensionnement du moteur électrique}

En premier lieu, les étudiants ont dû vérifier, par une étape de dimensionnement, que le moteur électrique a été correctement choisi. Cette phase du projet s'appuie entre autre sur un ouvrage regroupant l'ensemble des informations techniques (mécanique, électrique, paramétrage, ...) et pratiques (législation, coûts, ...) permettant à un particulier (ou à un groupe) de construire son propre véhicule électrique [17].

Les principales étapes du dimensionnement sont résumées dans la figure 3. Cinq phases sont à distinguer. La première nécessite de calculer la puissance motrice. Pour cela, le dimensionnement est mené à vitesse constante. Pour mener les calculs, les données suivantes sont nécessaires : la masse du véhicule (pilote inclus), les dimensions du châssis (largeur, hauteur), la largeur et la hauteur du pilote (pour estimer la surface de pénétration dans l'air), ainsi que le rendement du moteur.

Dans la deuxième phase du dimensionnement, il est important de disposer d'un profil de vitesse afin de calculer les forces accélératrice et décélératrice.

Enfin, il s'agit de calculer les couples moteur et résistant, de déterminer les quadrants de fonctionnement et choisir le moteur. 
Données:

- Masse du véhicule (pilote inclus)

- Dimensions du châssis :

- Largeur

- Hauteur

- Approximation de la surface de pénétration dans l'air :

- Hauteur du pilote

- Largeur du pilote

\section{Donnée :}

- Rendement du moteur

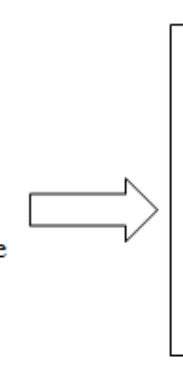

Calcul de la puissance motrice
Puissance résistive $=$ Force résistiv

Calcul de la puissance motrice
- Puissance résistive $=$ Force résistive $\times$ Vitesse

- Puissance motrice $=$ Puissance résistive / Rendement du moteur

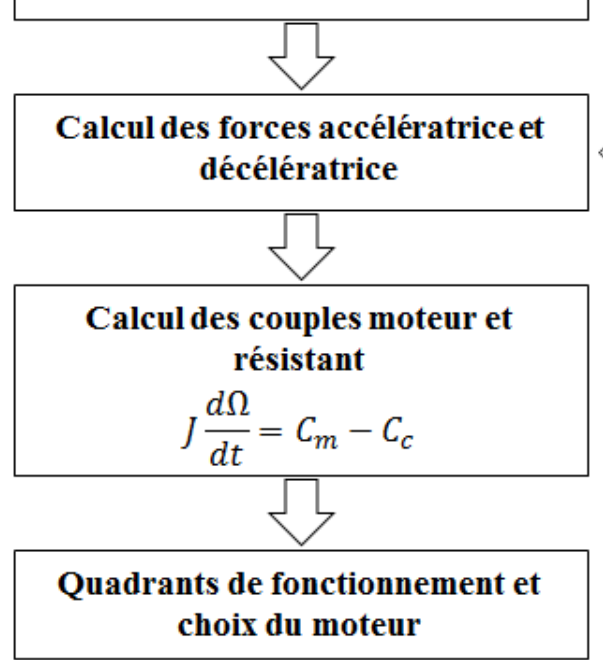
correspond à la force résistive
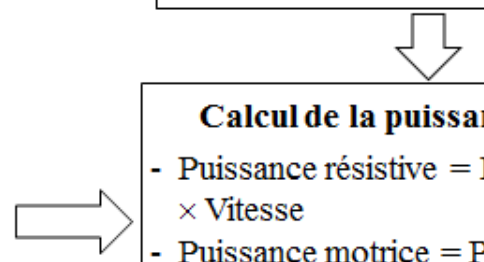

- Force de roulement

- Force aérodynamique (force $\rightarrow$ La somme de ces 2 forces

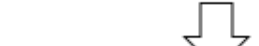

,
Dimensionnement à vitesse constante
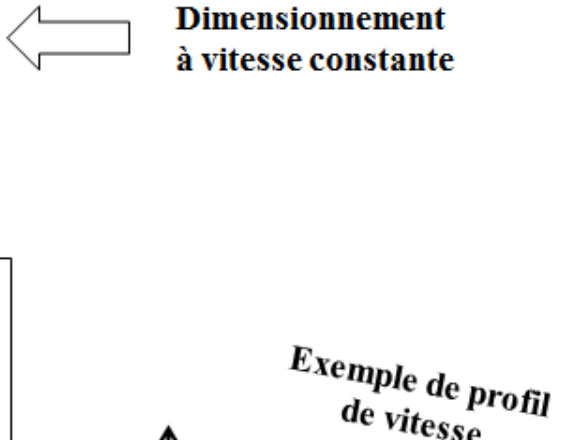

.

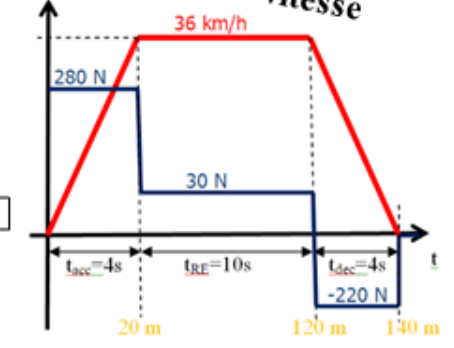

Vitesse $(\mathrm{km} / \mathrm{h})$ Forces électriques motrices ( $\mathrm{N}$ )

Figure 3 : Étapes de dimensionnement du moteur électrique.

\subsubsection{Aménagement du châssis}

La masse à vide du châssis du kart fourni au début du projet est de $100 \mathrm{~kg}$. Celui-ci est composé de contours tubulaires. Des pontons sont positionnés sur les côtés du véhicule permettant de le protéger, notamment en cas de chocs.

Comme l'indique la figure 4, les élèves ingénieurs ont tout d'abord retiré l'armature en acier, ainsi que les pontons. Le but est d'élargir le véhicule et de renforcer la base du châssis. Les étudiants ont ensuite fixé des plaques de polyéthylène à haute densité (PE-HD) d'un centimètre d'épaisseur. Ces plaques servent de support pour les batteries. Ce matériau trouve de nombreuses applications dont entre autre, les réservoirs de carburant pour automobile [19]. 


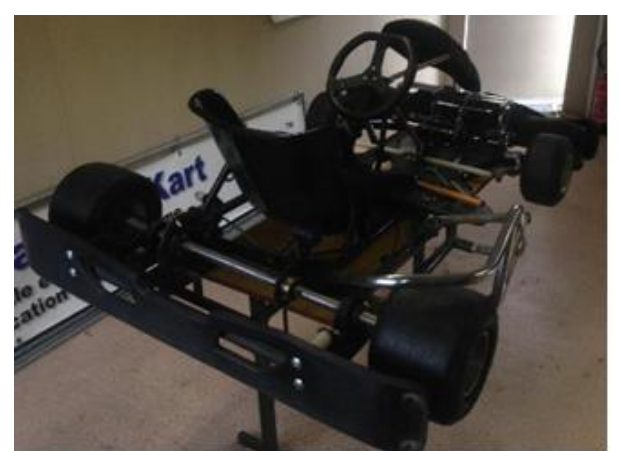

Allègement du châssis (retrait de l'armature en acier et des pontons)

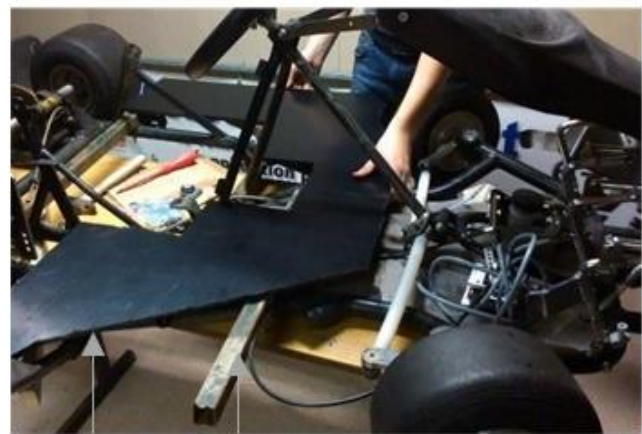

Élargissement et renforcement du châssis

Support des batteries (PE-

$\mathrm{HD}, 1 \mathrm{~cm}$ d'épaisseur)

Figure 4 : Aménagement du châssis du kart électrique.

\subsubsection{Fixation des éléments du système de motorisation et du tableau de bord}

La phase d'aménagement du châssis du kart étant achevée, les élèves ingénieurs ont procédé à la fixation des principaux éléments du système de motorisation électrique : les quatre batteries de $12 \mathrm{~V} 38 \mathrm{Ah}$, le système de branchement des chargeurs de batteries, le moteur synchrone triphasé «ME1304 PMSM Brushless » et le contrôleur électronique triphasé « SEVCON GEN4 G4845 » (cf. Figure 5). La sécurité électrique du véhicule est assurée par l'intermédiaire d'une protection contre les courts circuits, ainsi qu'un coupe-circuit général de type coup-de-poing d'arrêt d'urgence. Bien entendu, les élèves ingénieurs ont procédé au câblage électrique de tous ces éléments. Quant au tableau de bord, il est constitué d'un commutateur de Marche / Arrêt, d'un voyant lumineux indiquant que le kart électrique est sous tension et d'un commutateur Marche avant / Neutre / Marche arrière.

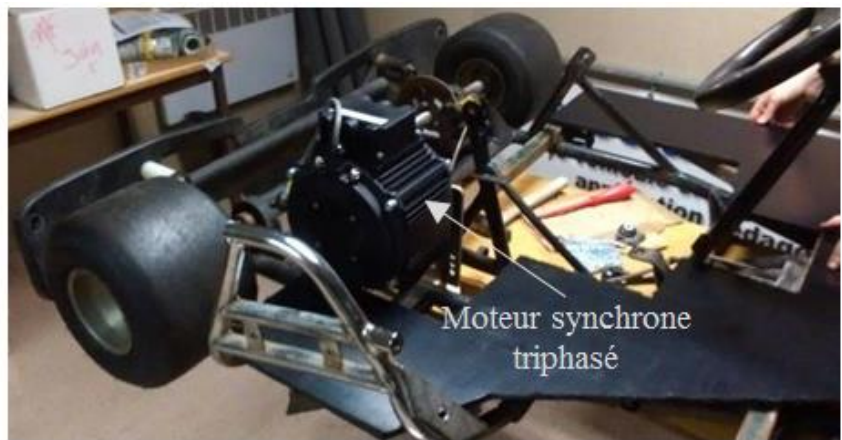

(a) Fixation du moteur synchrone triphasé «ME1304 PMSM Brushless »

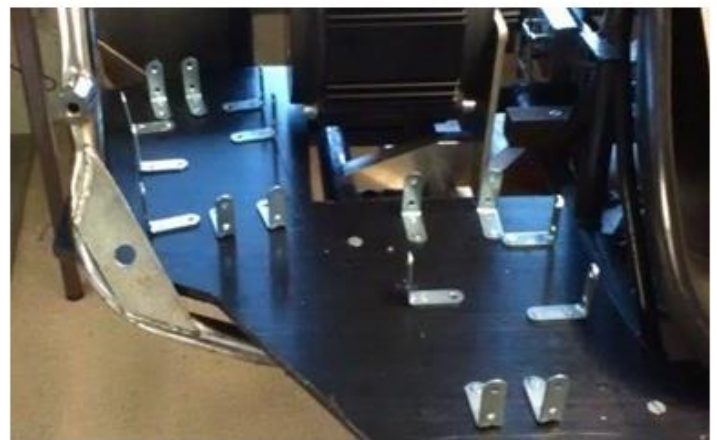

(b) Fixation des supports d'accueil des batteries 


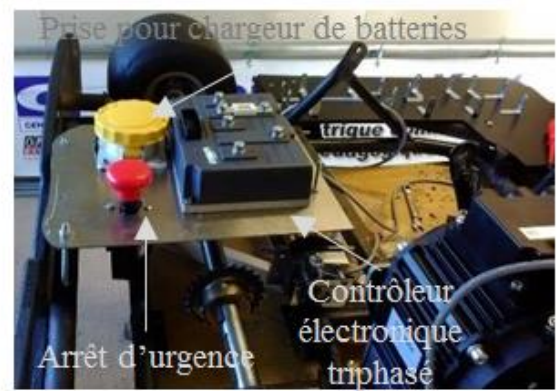

(c) Fixation du contrôleur « SEVCON GEN4 G4845 », du bouton d'arrêt d'urgence et de la prise du chargeur de batteries

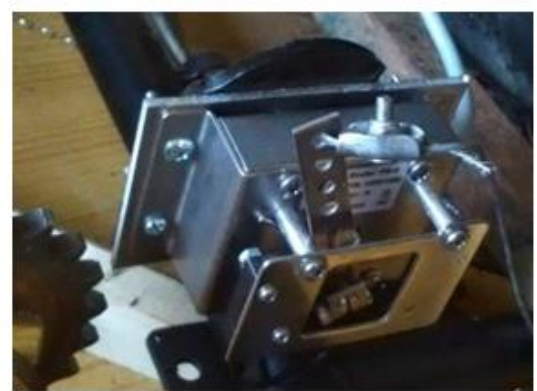

(d) Fixation du potentiomètre d'accélérateur

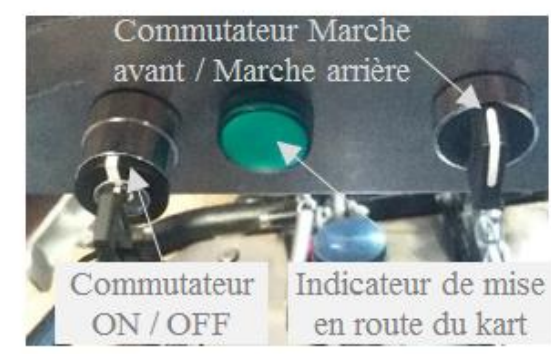

(e) Fixation du tableau de bord

Figure 5 : Fixation des éléments du système de motorisation et du tableau de bord.

\subsubsection{Un exemple d'innovation : récupération de l'énergie au freinage}

Dans le domaine de l'automobile, il est intéressant de récupérer une partie de l'énergie cinétique générée par le freinage d'un véhicule plutôt que de la disperser sous la forme de chaleur. Dans le domaine des sports mécaniques (e.g. Formule 1), des systèmes de récupération de l'énergie cinétique (SREC) ont été introduits dès 2009. Par l'usage du SREC, les pilotes peuvent utiliser l'énergie récupérée (400 kJ maximum par tour) en appuyant sur un bouton, déclenchant un afflux supplémentaire de puissance de 80 chevaux pendant 6,67 secondes (ou 40 chevaux pendant 13 secondes) dans les phases d'accélération [20-21].

Dans ce type d'application, le SREC peut s'appuyer sur un stockage inertiel tel qu'un volant d'inertie. Un tel système est composé d'un arbre de transmission qui se met en marche et actionne un embrayage qui, à son tour, actionne un autre arbre. Celui-ci fait tourner le disque contenu dans le volant d'inertie, puis l'embrayage est déconnecté du premier arbre. Lorsque l'on désire récupérer l'énergie, l'embrayage est connecté à un nouvel arbre qui est mis en rotation par l'énergie stockée dans le volant d'inertie. Le SREC par volant d'inertie présente 2 inconvénients majeurs : la masse élevée (près de $50 \mathrm{~kg}$ pour un SREC de cette nature associé à un moteur d'une puissance de 8,5 kW) du volant (le disque est généralement en acier) et son encombrement important.

Dans le cadre du projet collectif intensif, cette solution a été écartée puisque la masse et l'équilibre général du véhicule sont deux critères importants.

Le SREC proposé dans ce retour d'expériences est fondé sur l'utilisation de super-condensateurs. 
Il est important de rappeler que dans un super-condensateur tout comme une batterie, le stockage d'énergie électrique est possible lorsque le dispositif est en capacité de transférer et de stocker des ions ou des électrons. Pour ces deux dispositifs, l'électrolyte, composé d'un mélange d'ions positifs et négatifs, représente l'élément de base. Toutefois, deux différences fondamentales doit être notées. La première concerne la durée de recharge du dispositif de stockage et la seconde traite de sa capacité.

La durée de recharge est étroitement liée aux phénomènes physicochimiques. Dans une batterie, les réactions chimiques déplacent les ions de l'électrolyte vers l'intérieur ou en dehors de la structure atomique de la matière composant l'électrode. Cela induit un changement de degré d'oxydation du matériau en fonction de l'état de charge de la batterie. Dans le cas d'un super-condensateur, un champ électrique conduit les ions à se déplacer depuis (ou vers) la surface des électrodes sans réaction d'oxydoréduction. Comme les ions se fixent ou se détachent des électrodes sans aucune réaction chimique (capacité d'adsorption ou de désorption), un super-condensateur peut être chargé et déchargé très rapidement et cela, de façon répétée.

Quant à la capacité de stockage d'énergie électrique, celle-ci est plus importante pour une batterie puisqu'elle stocke les charges par réaction d'oxydoréduction dans le volume des matériaux. La quantité d'énergie stockée peut ainsi être conséquente. Ce n'est pas le cas d'un super-condensateur car il ne stocke les ions qu'à la surface de ses électrodes.

Les élèves ingénieurs ont procédé au dimensionnement de super-condensateurs. Pour cela, ils ont réglé un freinage électrique à $40 \%$. La figure 6 représente les résultats obtenus lors de cet essai. Avec un courant d'intensité maximale égale à $150 \mathrm{~A}$, une durée de $7 \mathrm{~s}$ et une variation de tension de $14 \mathrm{~V}$, la capacité aura pour valeur $75 \mathrm{~F}$. En d'autres termes, 75 Farads sont nécessaires pour récupérer $40 \%$ de l'énergie au freinage sous 64 V. Les étudiants ont alors installés sur le kart électrique 24 super-condensateurs MAXWELL (référence : BCAP3000 P270 K04) de 2,7 V DC et 3000 F (cf. Figure 7). Ces super-condensateurs sont associés en série. La capacité équivalente est de $125 \mathrm{~F}$ répondant ainsi au besoin de stockage d'énergie dans les phases de freinage du véhicule. Chaque composant pèse $510 \mathrm{~g}$. Certes ce type de SREC rajoute une masse sur le kart électrique de 12,2 kg, mais la gestion efficace des 24 super-condensateurs permettrait de prolonger d'au moins $20 \%$ l'autonomie des quatre 
batteries actuellement utilisées sur le véhicule.

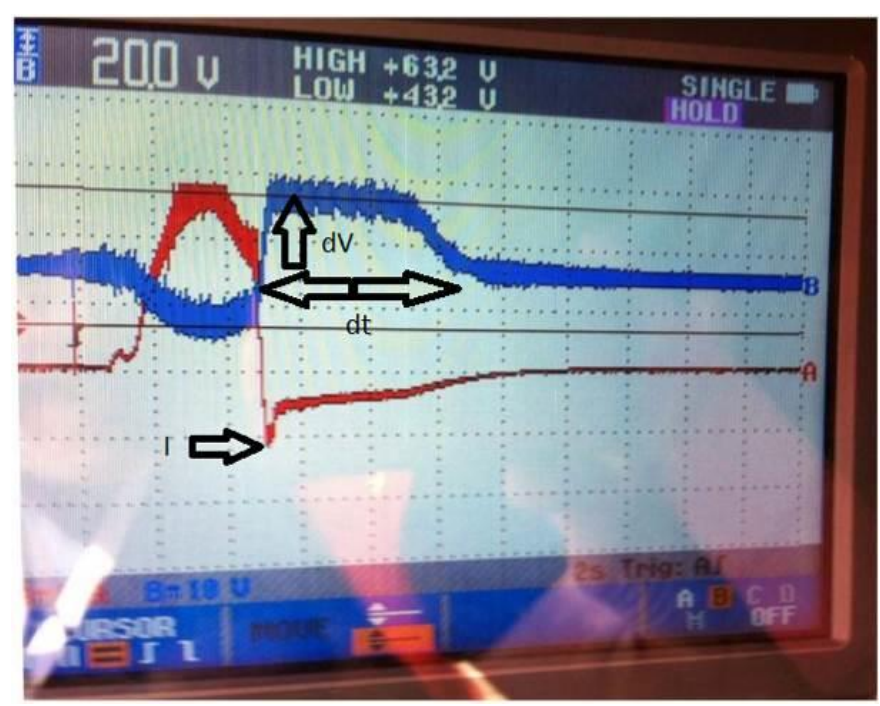

Figure 6: Relevé du courant et de la tension lors d'un freinage électrique à $40 \%$.

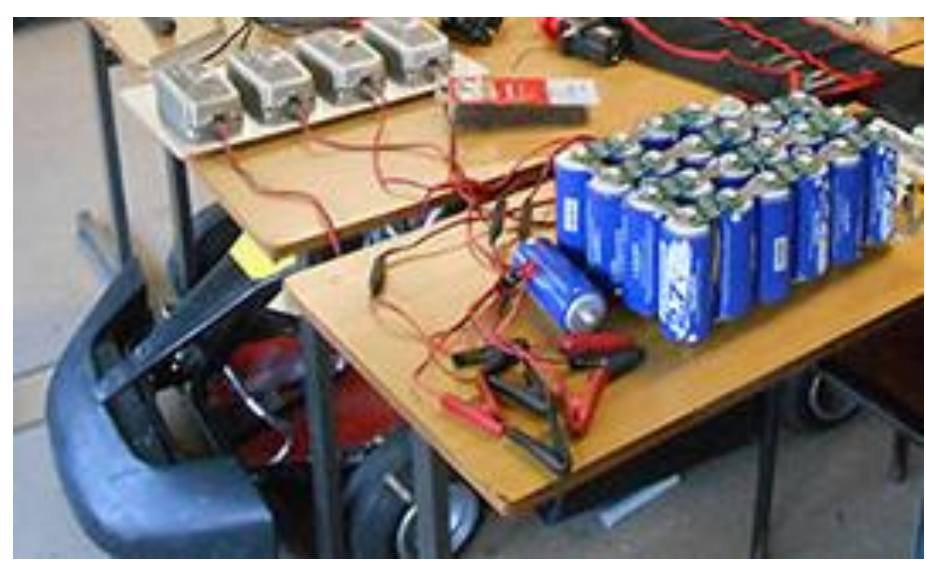

Figure 7 : Système de récupération de l'énergie cinétique au freinage à base de super-condensateurs.

Chaque super-condensateur est en mesure de fournir au maximum une tension de 2,7 V DC. La tension maximale obtenue par l'association en série de ces 24 dispositifs est alors de 64,8 V. Un convertisseur DC-DC abaisseur de tension est nécessaire puisque le système complet fonctionne en 48 V DC. Ce convertisseur n'a pas été réalisé durant ce projet par manque de temps. 


\section{Actions de communication}

Tous les élèves ingénieurs ont souhaité commenter en temps réel les moments clés des travaux menés dans le cadre du projet : définition des objectifs, des jalons et des livrables, dimensionnement et assemblage du véhicule, essais de validation du fonctionnement du kart électrique, actions de communication etc.

Pour cela, ils ont créé une page Facebook (https://www.facebook.com/Projet-Kart-Electrique-Polytech-ToursDEE-912424955459788). Toutes les informations ont également été relayées sur le site Internet de Polytech Tours (http://polytech.univ-tours.fr/polytech-tours/la-course-a-l-electrification-des-vehicules-502072.kjsp), ainsi que sur le site de l'association e-Kart (https://e-kart.fr/1307-polytech-tours-marathon-de-l-electrification-jours-7.html). Les élèves ingénieurs de $4^{\text {ème }}$ année de la spécialité « Électronique et Systèmes de l'Énergie Électrique » se sont enfin exercés au montage de spot publicitaire en vue de promouvoir leur formation. Par exemple, ils ont réalisé une vidéo d'une minute (https://www.youtube.com/watch?v=12n4PNHRpHM) mettant en exergue les spécificités de leur spécialité d'ingénieur. Dans ce type de spot publicitaire, le kart électrique est un excellent support pédagogique pluridisciplinaire qui permet d'illustrer les notions fondamentales et les compétences clés à acquérir par un étudiant.

Toutes ces actions de communication sont essentielles pour Polytech Tours puisqu'elles permettent d'accroître la lisibilité des formations d'ingénieurs associées. Elles permettent aussi de partager les principales réalisations avec le plus grand nombre de personnes, que ce soit en lien avec des établissements de formation (collèges, lycées, IUT, écoles d'ingénieurs), des passionnés du véhicule électrique etc.

Polytech Tours expérimente la démarche d'apprentissage par projet depuis quelques années. L'initiative pédagogique, mise en exergue dans cet article, porte sur une étude et réalisation d'un système industriel (un véhicule électrique performant) et à coût réduit (moins de 5000 Euros), toutes deux menées de manière à la fois collective et intensive (trois semaines consécutives réservées à l'aboutissement du projet).

Cette expérience a été largement saluée par la presse locale (La Nouvelle République, La Tribune Hebdo de Tours) et régionale (France 3 Régions). Un exemple est donné dans la figure 8. 


\section{Véhicules électriques: Polytech Tours se lance dans la course}
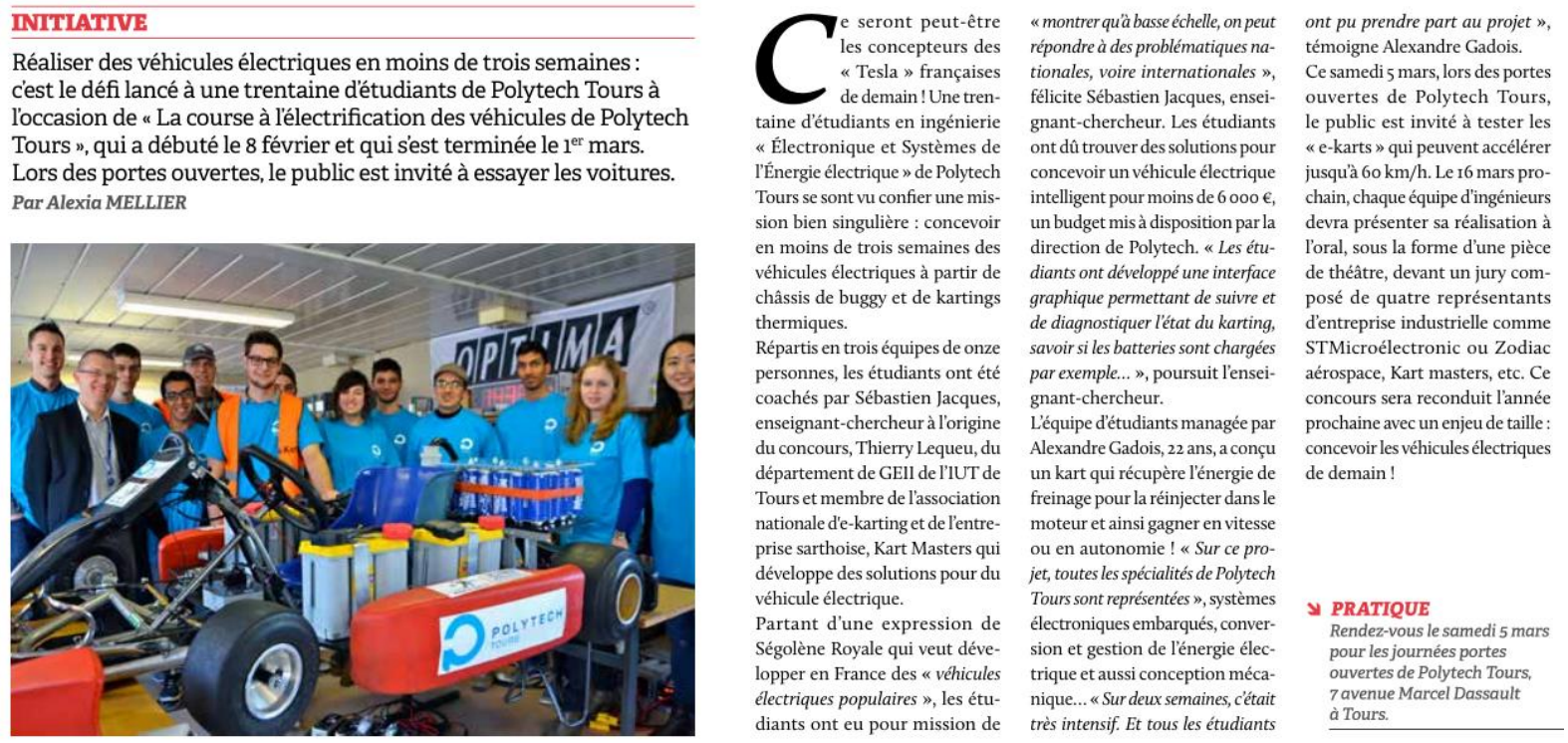

Figure 8: Projet collectif intensif autour du kart électrique salué par la presse locale (La Tribune Hebdo de

Tours, numéro 321).

\section{5. Évaluation du projet par les étudiants}

À l'issue de cette expérience collective, chacun des 33 étudiants a rempli un questionnaire dont le but est de donner une note de satisfaction globale du projet et en particulier, de son utilité dans la formation d'ingénieur.

Cinq questions ont été posées et notées sur une échelle de « 1 » « 4 ». Les notes « 1 » et « 4 » représentent respectivement les notes minimale et maximale obtenues pour chaque question. En particulier, les élèves ingénieurs ont pu donner leur avis sur plusieurs points : la pertinence du support technique utilisé (le kart électrique), le mode d'apprentissage des connaissances et des compétences (le projet collectif intensif) et l’organisation du projet (disponibilité du matériel, durée du projet, qualité de l'encadrement).

Le tableau 5 synthétise les résultats. Ce nouveau mode d'apprentissage a remporté un pourcentage de satisfaction de $83 \%$. 


\begin{tabular}{|c|c|c|c|c|c|c|}
\hline Question & $\begin{array}{l}\text { Pas du } \\
\text { tout }=1\end{array}$ & $\begin{array}{c}\text { Bien }= \\
2\end{array}$ & $\begin{array}{l}\text { Très Bien } \\
\quad=3\end{array}$ & $\begin{array}{l}\text { Excellent } \\
\quad=4\end{array}$ & Moyenne & Bilan \\
\hline $\begin{array}{l}\text { 1. Le kart électrique est-il un outil } \\
\text { pédagogique utile dans } \\
\text { l'acquisition des connaissances et } \\
\text { des compétences en conversion et } \\
\text { gestion de l'énergie électrique? }\end{array}$ & $1,4 \%$ & $12,4 \%$ & $39,6 \%$ & $46,6 \%$ & 3,31 & $82,8 \%$ \\
\hline $\begin{array}{l}\text { 2. Avez-vous le sentiment d'avoir } \\
\text { acquis davantage de connaissances } \\
\text { et de compétences par ce mode } \\
\text { d'apprentissage? }\end{array}$ & $3,1 \%$ & $13,1 \%$ & $40,6 \%$ & $43,2 \%$ & 3,24 & $81,0 \%$ \\
\hline $\begin{array}{l}\text { 3. Tous les éléments ont-ils été } \\
\text { réunis (disponibilité du matériel, } \\
\text { des encadrants, durée de } \\
\text { réalisation, ...) pour garantir le } \\
\text { succès du projet? }\end{array}$ & $2,8 \%$ & $8,9 \%$ & $60,2 \%$ & $28,1 \%$ & 3,14 & $78,4 \%$ \\
\hline $\begin{array}{l}\text { 4. Évaluer l'intérêt d'un projet } \\
\text { mené en groupe. }\end{array}$ & $1,2 \%$ & $6,5 \%$ & $35,3 \%$ & $57,0 \%$ & 3,48 & $87,0 \%$ \\
\hline $\begin{array}{l}\text { 5. Évaluer l'intérêt d'un projet } \\
\text { mené de façon intensive (plusieurs } \\
\text { semaines consécutives). }\end{array}$ & $1,2 \%$ & $7,5 \%$ & $38,5 \%$ & $52,8 \%$ & 3,43 & $85,7 \%$ \\
\hline Satisfaction globale & & & & & & $83,0 \%$ \\
\hline
\end{tabular}

Tableau 5 : Résumé des résultats de l'évaluation du projet collectif intensif.

\section{Conclusion}

Dans cet article, une nouvelle approche pédagogique autour du kart électrique a été expérimentée. Une trentaine d'élèves ingénieurs de quatrième année de la spécialité « Électronique et Systèmes de l’Énergie Électrique » de Polytech Tours, répartis en trois groupes, ont procédé à l'électrification d'un châssis de kart et proposé des solutions technologiques pour rendre le véhicule performant. Ce projet collectif a été mené de façon intensive. En effet, trois semaines consécutives à plein temps ont été dédiées à l'étude et à la réalisation du kart électrique. Durant les 56 heures encadrées, les étudiants ont justifié tous les éléments composant le système de motorisation (les batteries, le contrôleur, le moteur électrique), assemblé toutes les pièces du véhicule (partie mécanique et partie électrique) et validé le bon fonctionnement du kart. Ils ont également apporté une innovation technologique, notamment en récupérant l'énergie cinétique durant les phases de freinage du véhicule. Un système à base de super-condensateurs a été proposé. De nombreuses actions de communication ont été entreprises afin de valoriser 
cette initiative pédagogique ; initiative saluée par la presse locale et régionale.

Ce retour d'expériences permet de montrer que le kart électrique est un outil pédagogique pluridisciplinaire qui suscite l'apprentissage de savoirs (conception mécanique, systèmes électroniques embarqués, conversion et gestion de l'énergie électrique), de savoir-faire et de gestion de projet (décider, planifier, coordonner, ...).

\section{Bibliographie}

[1] M. J. Riezenman, "Engineering the EV future", IEEE Spectrum, Vol. 35, Issue 11 (1998), pp. 18-20.

[2] J. Lassila, J. Haakana, V. Tikka, J. Partanen, "Methodology to Analyze the Economic Effects of Electric Cars as Energy Storages", IEEE Transactions on Smart Grid, Vol. 3, Issue 1 (2012), pp. 506-516.

[3] E. Labeye, J. Adrian, M. Hugot, M. A. Regan, C. Brusque, "Daily use of an electric vehicle: behavioural changes and potential for its support", IET Intelligent Transport Systems, Vol. 7, Issue 2 (2013), pp. 210-214.

[4] R. T. M. Smokers, M. Verbeek, S. van Zyl, "EVs and post 2020 CO2 targets for passenger cars", Proceeding of the 2013 World Electric Vehicle Symposium and Exhibition, 2013, pp. 1-11.

[5] A. Sanchez-Miralles, T. Gomez San Roman, I. J. Fernandez, C. F. Calvillo, "Business Models Towards the Effective Integration of Electric Vehicles in the Grid", IEEE Intelligent Transportation Systems Magazine (2014), pp. 45-56.

[6] M. Torregrossa, "Véhicule électrique \& Analyse du Cycle de Vie - L'ADEME confirme la pertinence environnementale du VE", Association avem $1^{\text {er }}$ site d'information sur le véhicule électrique et hybride, http://avem.fr/actualite-vehicule-electrique-et-analyse-du-cycle-de-vie-l-ademe-confirme-la-pertinenceenvironnementale-du-ve-4637.html, Posté le 13/12/2013.

[7] "Plus de 100000 véhicules électriques immatriculés dans le monde en 2015", Avere France - Association nationale pour le développement de la mobilité électrique, http://www.averefrance.org/Site/Article/?article_id=6267, Posté le 07/09/2015.

[8] F. Carranza, O. Paturet, S. Salera, "Norway, the most successful market for electric vehicles", Proceeding of the 2013 World Electric Vehicle Symposium and Exhibition, 2013, pp. 1-6.

[9] P. Haugneland, H. H. Kvisle, "Norwegian electric car user experiences", Proceeding of the 2013 World 
Electric Vehicle Symposium and Exhibition, 2013, pp. 1-11.

[10] M. N. Eisler, "A Tesla in every garage?", IEEE Spectrum, Vol. 53, Issue 2 (2016), pp. 34-55.

[11] A.-G. Verdevoye, "Renault-Nissan: comment Carlos Ghosn s'est trompé sur l'électrique", Challenges économie - Auto, http://automobile.challenges.fr/actu-auto/20150625.CHA7306/renault-nissan-comment-carlosghosn-s-est-trompe-sur-l-electrique.html, Publié le 25/06/2015.

[12] R. Heuillard, "COP21 : vers des voitures électriques à moins de 7000 euros ", Clubic Mag - Transports, http://www.clubic.com/mag/transports/actualite-788544-cop21-voiture-electrique-de-5000-a-7000-euros.html, Publié le 04/12/2015.

[13] T. Lequeu, B. Bidoggia, Y. Derrien, N. Godefroy, "Two examples of pedagogical applications of electrical go-karts ", Proceeding of the 2007 European Conference on Power Electronics and Applications, 2007, pp. 1-9. [14] T. Lequeu, B. Bidoggia, A. Schellmanns, Y. Derrien, N. Godefroy, "Exemples d'applications pédagogiques autour du kart électrique e-kart", J3eA (2008).

[15] A. Sivert, F. Betin, T. Lequeu, "Réalisation d'un kart électrique performant : gestion de l'énergie embarquée et choix technologiques", J3eA (2015).

[16] A. Sivert, F. Betin, T. Lequeu, F. Maeght, "Pedagogical study of electric go-karts: Technological choices, instrumentations, characteristics, challenge", WSEAS Transactions on Advances in Engineering Education, Vol. 12 (2015), pp. 95-104.

[17] A. Sivert, T. Lequeu, "Je construis mon véhicule électrique—Vélos, karts et motos", Dunod, Collection ETSF, 2013, 144 p.

[18] H. Ibrahim, A. Ilinca, J. Perron, "Energy storage systems - Characteristics and comparisons", Renewable and Sustainable Energy Reviews, Vol. 12, Issue 5 (2008), pp. 1221-1250.

[19] C. Penu, "Polyéthylène haute densité PE-HD - Contrôle", Techniques de l'ingénieur, Référence AM3315 (2011).

[20] G. Richards, "It's green for go", Engineering \& Technology, Vol. 3, Issue 20 (2008), pp. 44-47.

[21] D. Cross, J. Hilton, "High Speed Flywheel Based Hybrid Systems for Low Carbon Vehicles", Proceeding of 
the 2008 Hybrid and Eco-Friendly Vehicle Conference, 2008, pp. 1-5.

Sébastien Jacques a obtenu un diplôme d'Ingénieur en Électronique et Productique, ainsi qu'un Master Recherche en Électronique, Signal et Microsystèmes à l'Université de Tours en 2007 et le Doctorat d'électronique de l'Université de Tours en 2010. Il a effectué sa thèse au laboratoire LMP (Laboratoire de Microélectronique de Puissance) à Tours. Après deux années en tant qu'Ingénieur Fiabilité Produits au sein du centre STMicroelectronics de Tours, il a rejoint en 2012, en tant que Maître de Conférences, le GREMAN (Groupe de Recherche Matériaux, microélectronique, Acoustique, Nanotechnologies) UMR-CNRS 7347 et Polytech Tours où il enseigne principalement l'électronique de puissance et plus particulièrement, les systèmes de conversion et de gestion de l'énergie électrique. Ses recherches portent sur les composants de puissance, leur intégration dans des systèmes de conversion d'énergie électrique et leur fiabilité.

Thierry Lequeu est Maître de conférences de génie électrique. Il enseigne l'électronique de puissance au sein du département Génie Électrique et Informatique Industrielle (GEII) de l'IUT de Tours. Depuis 2006, il est Président de l'association e-Kart et organise tous les ans le Challenge International Pédagogique de kart électrique e-Kart.

Valéry Dewancker est gérant de la société Kart Masters. Il participe activement à l'organisation d'événements pédagogiques autour du véhicule électrique et apporte un soutien technique aux établissements de formation désirant utiliser le véhicule électrique comme support pédagogique pluridisciplinaire. 